\title{
Effects of melatonin implantation during the slow period of cashmere growth on fibre production
}

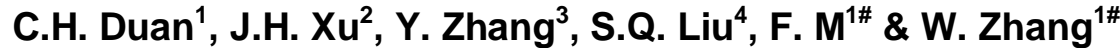 \\ ${ }^{1}$ State Key Laboratory of Animal Nutrition, College of Animal Science and Technology, China Agricultural University, \\ Beijing 100193, P. R. China \\ ${ }^{2}$ College of Veterinary Medicine, China Agricultural University, Beijing 100193, P. R. China \\ ${ }^{3}$ Centre for Animal Welfare and Ethics, University of Queensland, Gatton 4343, Brisbane, Queensland, Australia \\ ${ }^{4}$ Inner Mongolia YiWei White Cashmere Goat Farm, E'erduoshi, Inner Mongolia 016100, P.R. China
}

(Received 5 November 2015; Accepted 19 May 2016; First published online 28 June 2016)

Copyright resides with the authors in terms of the Creative Commons Attribution 2.5 South African Licence.
See: http://creativecommons. org/licenses/by/2.5/za
Condition of use: The user may copy, distribute, transmit and adapt the work, but must recognise the authors and the South African
Journal of Animal Science.

\begin{abstract}
This study was conducted to investigate the effects of melatonin implantation during the slow period of cashmere growth on fibre production in Inner Mongolian cashmere goats. It was found that melatonin implantation had no effect on the growth rate of cashmere, except from February to March when the rate of treated goats $(0.16 \mathrm{~mm} / \mathrm{d})$ was significantly higher than that of control goats $(0.06 \mathrm{~mm} / \mathrm{d})$. However, although melatonin administration delayed the rate of decline in cashmere growth rate for one month, there was no significant increase in cashmere production in melatonin-treated goats. It is evident that melatonin implantation during the slow period of growth cannot be used to prolong cashmere growth in Inner Mongolian cashmere goats.
\end{abstract}

Keywords: Cashmere yield, fibre characteristics, growth rate, reproductive performance

\# Corresponding author: mofang@cau.edu.cn, wzhang@cau.edu.cn

\section{Introduction}

The Inner Mongolian cashmere goat is one of the major cashmere goat breeds in China, with an average cashmere fibre diameter under $15 \mu \mathrm{m}$ and an average fibre length of $4-8 \mathrm{~cm}$ (China National Commission of Animal Genetic Resources, 2011). Seasonal cashmere growth cycles in Inner Mongolian cashmere goats have been well defined. They start in August and cease the following February, with the fleece being shed at the end of April (Da et al., 1991). The fast-growing period for cashmere in the goats occurs from August to December, the slow period from January to February and the non-growing period from March to July (Zhang, 2011).

A number of studies have shown that cashmere growth can be initiated by administering melatonin during the non-growing period (Betteridge et al., 1987; Klören \& Norton, 1995; Wang et al., 2006). In a recent experiment it was demonstrated that administration of melatonin $(2 \mathrm{mg} / \mathrm{kg}$ bodyweight (BW)) during the non-growing period increased cashmere yield by $34.5 \%$ and length by $21.3 \%$ (Duan et al., 2015). These increases were attributed to an extension in the duration of growth by combining a period of growth as a result of melatonin administration with the normal period of growth.

Melatonin treatment during the slow period has been used in an attempt to delay the onset of fibre shedding (Mitchell et al., 1991; Dicks et al., 1995; Klören \& Norton, 1995; Cong et al., 2011). Mitchell et al. (1991) found no detectable delay in shedding cashmere, while Dicks et al. (1995) demonstrated that melatonin implantation caused an advance, rather than a delay, in the onset of the spring moult. In addition, Klören \& Norton (1995) reported that melatonin implantation from January to April reduced the maximum fleece length compared with controls by causing an early cessation of growth. In contrast, Cong et al. (2011) reported that cashmere continued to grow until June in goats treated with melatonin, whereas the growth ceased in March in control goats. However, the effects of melatonin during the slow period have not been determined in Inner Mongolian cashmere goats.

The winter solstice is the turning point when cashmere growth changes from fast to slow. Therefore, the objective of the current study was to examine the effects of melatonin administration from December (winter solstice) to April on the cashmere growth cycle in Inner Mongolian cashmere goats. In particular, this study examined whether such treatment could effectively improve cashmere growth and prolong it. 


\section{Materials and Methods}

This experiment was conducted at YiWei White Cashmere Goat Farm in the Inner Mongolia Autonomous Region of China (latitude $39^{\circ} 06^{\prime} \mathrm{N}$, longitude $107^{\circ} 59^{\prime} \mathrm{E}$, altitude $1,500 \mathrm{~m}$ ) from 26 April 2013 to 26 April 2014. All procedures used in this study were approved by the Animal Care and Use Committee of China Agricultural University (Beijing, China).

The feeding and management of the goats were typical of those of other goats at the cashmere goat farm where goats are kept year round on desert pasture with occasional supplementary feeding. The grazing and supplementary feeding management of the goats have been described (Duan et al., 2015). Mating occurred over 30 - 60 days (October - November, 2013) with parturition in March - April 2014.

Thirty female Inner Mongolian cashmere goats (three years old with an initial bodyweight of $36.3 \pm 1.43$ $\mathrm{kg}$ ) were combed with a small rake on 26 April 2013 to remove the previous year's fleece. None of the goats had previously received any hormonal treatment or been exposed to an artificial photoperiod.

The goats were randomly assigned to one of two groups $(n=15)$, namely a control and a treatment group. The treated goats received subcutaneous melatonin implants (Beijing Kangtai Biological Technology Company, Beijing, China) at the base of the ear on 22 December 2013 and 22 February 2014 to provide a continuous release of melatonin. All goats were pregnant when melatonin was implanted in December. The implants were made from medical grade silicone elastomer, and were designed to release melatonin slowly for at least 60 days (manufacturer's specifications). Treated goats received $2 \mathrm{mg}$ melatonin/kg bodyweight and the dose was based on the research of Yue et al. (2007).

Starting in December and subsequently at one-month intervals until April, a $30 \times 30 \mathrm{~mm}$ patch of fleece was shorn from the mid side of each goat at skin level for subsequent analyses of fibre length and diameter. Fleece sampling was conducted according to the method of Duan et al. (2015). The cashmere growth length of each month was calculated by the subtraction method. Cashmere was harvested on 26 April 2014 by combing, as described above, and was weighed with an electronic scale. Therefore, the average length of the cashmere fibre samples in April was the maximum, and is one of the fibre characteristics that may represent the cashmere production.

The samples were soaked in carbon tetrachloride detergent solution overnight, rinsed thoroughly in deionized water, and dried at $80^{\circ} \mathrm{C}$. One hundred cashmere fibres were randomly chosen from each sampling date of each goat to measure the stretched length of the cashmere with a scaled ruler. The diameter of 200 randomly chosen fibre samples was measured with an optic fibre diameter analyser (CU-6, Beijing United Vision Technical Company, Beijing, China).

The data were analysed with the software package SAS (SAS, 2009). Differences between mean values of melatonin-treated goats and control goats were obtained using the unpaired Student's t-test. Differences were considered statistically significant at the levels of $P<0.05$.

\section{Results}

Cashmere fibre growth for the treatment group was similar to that of the control group (Figure 1). The slopes and close proximity of the two curves show no evidence that the period of cashmere growth was prolonged by melatonin treatment from December to April. However, melatonin implantation delayed the rate of decline in cashmere growth from February to March.

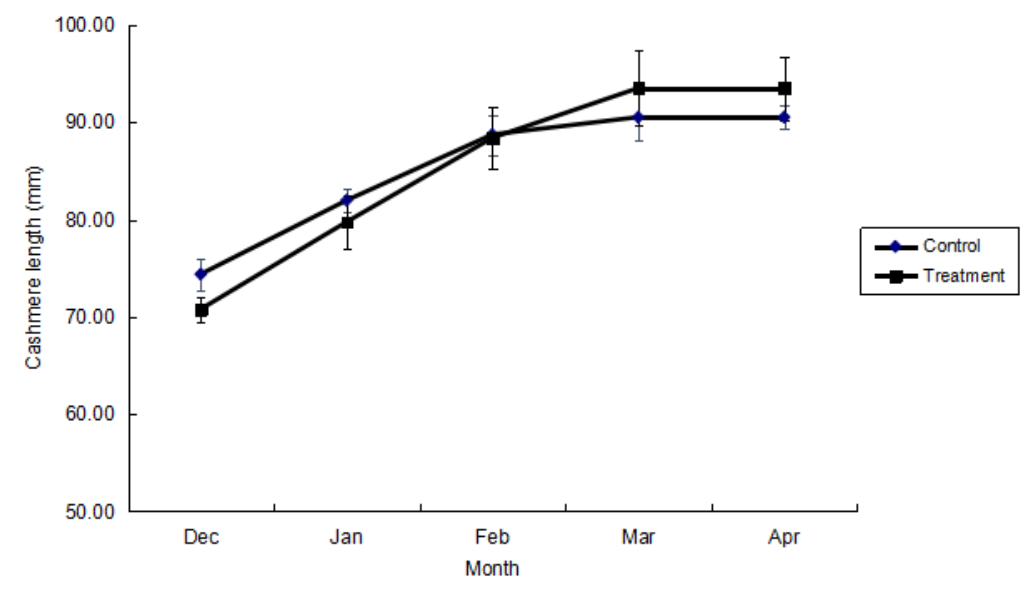

Figure 1 Cumulative cashmere length $(\mathrm{mm})$ for control and melatonin implanted goats (from December to April). Values are means and standard error of 15 replicates per treatment. 
Cashmere growth slowed from December onwards in both the control and melatonin-treated goats (Table 1). No significant differences were observed in cashmere growth rate between treated and control goats, except from February to March, when the growth rate of melatonin-treated goats $(0.16 \mathrm{~mm} / \mathrm{d})$ was significantly $(P<0.03)$ higher than that of the control goats $(0.06 \mathrm{~mm} / \mathrm{d})$. No cashmere growth was observed $(0$ $\mathrm{mm} / \mathrm{d}$ ) in either the control or the melatonin-treated goats from March to April.

The effects of melatonin treatment on goat performance are shown in Table 2. All goats gained weight over the course of the experiment. There was no significant effect of melatonin treatment on mean live weight or average daily gain. Nor were significant differences observed in cashmere yield, fibre diameter and maximum length of growth between the control and melatonin-treated goats $(P>0.05)$.

Table 1 Mean cashmere fibre growth rate $(\mathrm{mm} / \mathrm{d})$ in melatonin-treated and control goats from December to April $^{1}$

\begin{tabular}{lcccc}
\hline \multirow{2}{*}{ Group } & \multicolumn{3}{c}{ Month } \\
\cline { 2 - 5 } & $\begin{array}{l}\text { December to } \\
\text { January }\end{array}$ & $\begin{array}{c}\text { January to } \\
\text { February }\end{array}$ & $\begin{array}{c}\text { February to } \\
\text { March }\end{array}$ & $\begin{array}{c}\text { March to } \\
\text { April }\end{array}$ \\
\hline Control & & & & \\
Melatonin & 0.30 & 0.20 & $0.06^{\mathrm{b}}$ & 0 \\
SEM & 0.35 & 0.27 & $0.16^{\mathrm{a}}$ & 0 \\
$P$-value & 0.07 & 0.05 & 0.04 & 0 \\
& 0.48 & 0.30 & 0.02 & --
\end{tabular}

\footnotetext{
${ }^{1}$ Values are means of 15 replicates per treatment. Means with different superscripts ( $a$ and $b$ ) within a column are significantly different at $P<0.05$.

SEM: standard error of mean.
}

Table 2 Effect of melatonin implantation on bodyweight gain and cashmere production of Inner Mongolian cashmere goats $^{1}$

\begin{tabular}{|c|c|c|c|c|c|c|}
\hline \multirow[b]{2}{*}{ Group } & \multicolumn{2}{|c|}{${\text { Live weight }(\mathrm{kg})^{2}}^{2}$} & \multirow{2}{*}{$\begin{array}{l}\text { Average daily } \\
\text { gain (g) }\end{array}$} & \multicolumn{3}{|c|}{ Cashmere production } \\
\hline & $\begin{array}{c}\text { Initial } \\
\text { bodyweight }\end{array}$ & $\begin{array}{c}\begin{array}{c}\text { Final } \\
\text { bodyweight }\end{array} \\
\end{array}$ & & $\begin{array}{c}\text { Cashmere yield } \\
\text { (g) }\end{array}$ & $\begin{array}{c}\text { Maximum length } \\
(\mathrm{mm})\end{array}$ & $\begin{array}{c}\text { Cashmere fibre } \\
\text { diameter }(\mu \mathrm{m})\end{array}$ \\
\hline Control & 35.8 & 38.9 & 8.45 & 635.0 & 90.5 & 14.5 \\
\hline Melatonin & 37.0 & 39.5 & 6.85 & 665.8 & 93.5 & 14.2 \\
\hline SEM & 1.43 & 1.90 & 3.32 & 53.4 & 3.46 & 0.24 \\
\hline$P$-value & 0.42 & 0.76 & 0.64 & 0.57 & 0.42 & 0.21 \\
\hline
\end{tabular}

\footnotetext{
${ }^{1}$ Values are means of 15 replicates per treatment.

${ }^{2}$ Initial bodyweight was taken when cashmere production ceased in April 2013 and final bodyweight when cashmere production ceased in April 2014.

SEM: standard error of mean.
}

The effects of melatonin treatment on reproductive performance are presented in Table 3 . The kidding date, litter size and birth weight did not differ significantly between the control and melatonin-treated goats $(P>0.05)$.

\section{Discussion}

The results of this study demonstrate that melatonin implantation of Inner Mongolian cashmere goats from December to April did not prolong cashmere growth to the extent that was reported for Liaoning cashmere goats (Cong et al., 2011), nor did it cause an advance in the onset of the spring moult, as was reported in adult feral $\times$ dairy-type female goats (Dicks et al., 1995) and Australian cashmere goats (Klören \& Norton, 1995). 
Table 3 Effects of melatonin implantation on the reproductive performance of Inner Mongolian cashmere goats $^{1}$

\begin{tabular}{lcccc}
\hline \multirow{2}{*}{ Item } & \multicolumn{2}{c}{ Treatment } & \multirow{2}{*}{ SEM } & \multirow{2}{*}{ P-value } \\
\cline { 2 - 3 } & Control & Melatonin & & \\
\hline Kidding date $^{*}$ March 18, 2014 & March 19, 2014 & 3.07 & 0.65 \\
Litter size $^{2}$ & 1.29 & 1.11 & 0.12 & 0.16 \\
Birth weight (kg) $^{2.99}$ & 3.00 & 0.17 & 0.96 \\
\hline
\end{tabular}

\footnotetext{
${ }^{1}$ Values are means of 15 replicates per treatment.

${ }^{2}$ Litter size $=$ mean number of kids per birth.

SEM: standard error of mean.
}

Growth of cashmere exhibits a seasonal rhythm arising from circannual changes in the natural photoperiod, with growth beginning after the longest day of the year (summer solstice) and moult occurring after the winter solstice (Klören \& Norton, 1993; Dicks et al., 1994; Santiago-Moreno et al., 2004). Similarly, in the current study, cashmere growth slowed from December (winter solstice) onwards and cashmere shedding occurred in April in both treatments. Administration of melatonin from December to April improved cashmere growth only from February to March. Despite this, the extra growth was insufficient to result in greater cumulative cashmere growth during the entire trial period. Therefore, melatonin administered from December to April offered no practical advantage in prolonging the period of cashmere growth or delaying shedding.

Similarly, Mitchell et al. (1991) reported that melatonin implantation (125 mg; implant insertion every two months) of reproducing cashmere-bearing female New Zealand cashmere goats (over two years old) in late autumn and winter did not delay the date of shedding. However, in the research of Dicks et al. (1995), 10 juvenile ( 8 months old) and 10 adult feral $\times$ dairy-type female cashmere goats received continuous release melatonin implants, $18 \mathrm{mg}$ on 11 December, 1 February and 1 April. It demonstrated that at least in the United Kingdom, melatonin administration cannot be used to delay the onset of the spring moult. It has the opposite effect in adult goats and none in juvenile goats.

In a study by Klören \& Norton (1995), four male and four female Australian cashmere goats (10 months old) were implanted with melatonin $(18 \mathrm{mg})$ at monthly intervals for three months, commencing in January. Their results indicated that the administration of melatonin reduced the maximum fleece length compared with the control, which was caused by an early cessation of cashmere growth.

In contrast, Cong et al. (2011) concluded that melatonin implantation $(2 \mathrm{mg} / \mathrm{kg} \mathrm{BW}$ with implantation every two months) of 16 castrated Liaoning cashmere goats (one year old, with initial bodyweight of $30 \mathrm{~kg}$ ) from December to June successfully extended the cashmere growth phase for three months. The discrepancies among studies on the effects of melatonin implantation during the cashmere slow period on cashmere growth may be due to differences in breed, sex, age of goats and the dose and duration of melatonin treatment.

Similar to the studies of Dicks et al. (1995) and Cong et al. (2011), the results of the current study showed no significant effects of melatonin implantation on live weight and average daily gain, which indicates that melatonin implantation during the slow period did not affect the growth of Inner Mongolian cashmere goats. Although melatonin administration delayed the rate of decline in cashmere growth rate during the slow period, there was no significant increase in cashmere production, which is in accordance with the findings of Mitchell et al. (1991).

The effect of melatonin implantation on cashmere fibre diameter has not been consistent. During the slow period, Cong et al. (2011) reported that implantation of melatonin had no effect on fibre diameter in Liaoning cashmere goats. Similarly, cashmere fibre diameter was not affected by melatonin implantation in the current study. Duan et al. (2015) reported that melatonin implantation during the cashmere non-growing period significantly decreased cashmere fibre diameter in Inner Mongolian cashmere goats. Based on an experiment conducted for one year, Chang (2010) concluded that administration of melatonin had no effect on fibre diameter in Inner Mongolian cashmere goats.

Some experiments have shown that melatonin implantation over a period of time increased the fibre diameter in New Zealand cashmere goats (Moore et al., 1989) and Spanish goats (Wuliji et al., 2003). However, other experiments found that administration of melatonin had no effects on fibre diameter in New Zealand cashmere goats (O'Neill et al., 1992) and Inner Mongolian cashmere goats (Yue et al., 2007). The discrepancy in the results for fibre diameter in these reports may be related to differences in goat breed and 
the time of melatonin treatment.

Reproductive activity in goats shows an annual pattern, with long days inhibiting ovarian activity and short days stimulating it (Zarazaga et al., 2011). The evidence suggests that as day length decreases during autumn, an increase in the duration of secretion of melatonin triggers an endocrine response, leading to the onset of reproductive activity (Chemineau et al., 1987).

Kumar \& Purohit (2009) reported that melatonin administration could initiate the breeding season one to two months earlier in goats. This could be advantageous when using accelerated breeding systems. However, relatively few reports have been published on the effect of melatonin implantation on reproductive performance in cashmere goats. Chang (2010) reported that continuous melatonin treatment for a year did not exert any significant effect on reproductive performance in Inner Mongolian cashmere goats. Similarly, Duan et al. (2015) reported that melatonin implantation during the anoestrous season did not affect the reproductive performance of Inner Mongolian cashmere goats in the following year. The results of the current study showed similar findings in that reproductive performance was not affected by melatonin implantation from December to April, which implies that melatonin implantation during gestation did not affect the reproductive performance of cashmere goats.

Melatonin administration during the non-growing period (Duan et al., 2015) and the slow period for cashmere growth (the present study) was studied in an attempt to induce cashmere growth ahead of schedule and improve it by delaying shedding in Inner Mongolian cashmere goats. It appears that the effects of melatonin administration during the non-growing period for cashmere are far greater than during the slow period.

\section{Conclusion}

Melatonin administration ( $2 \mathrm{mg} / \mathrm{kg} \mathrm{BW}$ ) during the slow period (December to April) increased cashmere growth rate only from February to March without affecting average daily gain or reproductive performance. Melatonin implantation during the slow period did not increase production or prolong the period of cashmere growth.

\section{Acknowledgements}

This work was supported by the Key Programme of the National Natural Science Foundation of China (No. 31470119) and the China Agriculture Research System (CARS-40).

\section{Authors' Contributions}

CHD conceived of the study, carried out the experiments and drafted the manuscript. JHX and YZ assisted with the sample analysis. FM, SQL and WZ participated in the study's design and coordination. All authors read and approved the final manuscript.

\section{Conflict of Interest Declaration}

The authors declare that they have no competing interests.

\section{References}

Betteridge, K., Welch, R.A.S., Pomroy, W.E., Lapwood, K.P. \& Devantier, B.P., 1987. Out of season cashmere growth in feral goats. In: Proceedings of the Second International Cashmere Conference, Lincoln College, New Zealand. pp. 137-142.

Chang, Z.L., 2010. Study on effect of constant-release melatonin implants on the cashmere growth traits of cashmere goats and related gene. Ph.D. thesis, Inner Mongolia Agricultural University, Hothot, Inner Mongolia, China. (in Chinese, English abstract).

Chemineau, P., Daveau, A., Maurice, F. \& Thimonier, J., 1987. Seasonality and light control of reproduction in goat. Proceeding of the 38th Annual Meeting of the European Association in Animal Production, Lisbon, Portugal. p. 906.

China National Commission of Animal Genetic Resources, 2011. Animal Genetic Resources in China: Sheep and Goats. China Agricultural Press, Beijing. (in Chinese).

Cong, Y.Y., Deng, H.W., Feng, Y.L., Chen, Q. \& Sun, Y., 2011. Melatonin implantation from winter solstice could extend the cashmere growth phase effectively. Small Rumin. Res. 99, 48-53.

Da, W.Z., Liu, Z.X., Zhao, H., Wang, T.X., Kang, J., Yu, Y. \& Yang, X., 1991. The determination of cashmere growth period. Chin. Herb. Sci. 2, 45-46. (in Chinese).

Dicks, P., Russel, A. \& Lincoln, G.A., 1994. The role of prolactin in the reactivation of hair follicles in relation to moulting in cashmere goats. J. Endocrinol. 143, 441-448.

Dicks, P., Russel, A. \& Lincoln, G.A., 1995. The effect of melatonin implants administered from December until April on plasma prolactin, triiodothyronine and thyroxine concentrations and on the timing of the spring moult in cashmere goats. Anim. Sci. 60, 239-247. 
Duan, C.H., Xu, J.H., Sun, C.M., Jia, Z.H. \& Zhang, W., 2015. Effects of melatonin implantation on cashmere yield, fibre characteristics, duration of cashmere growth as well as growth and reproductive performance of Inner Mongolian cashmere goats. J. Anim. Sci. Biotechnol. 6, 463-468.

Klören, W.R.L. \& Norton, B.W., 1993. Fleece growth in Australian cashmere goats: IV. The role of prolactin in the initiation and cessation of cashmere growth. Crop Pasture Sci. 44, 1051-1061.

Klören, W.R.L. \& Norton, B.W., 1995. Melatonin and fleece growth in Australian cashmere goats. Small Rumin. Res. 17, 179-185.

Kumar, S. \& Purohit, G.N., 2009. Effect of a single subcutaneous injection of melatonin on estrous response and conception rate in goats. Small Rumin. Res. 82, 152-155.

Mitchell, R.J., Betteridge, K., Gurnsey, M.P. \& Welch, R.A.S., 1991. Fibre growth of cashmere-bearing goats given melatonin in late autumn and winter. New Zeal. J. Agric. Res. 34, 419-425.

Moore, R.W., Bigham, M.L. \& Staples, L.D., 1989. Effect of Regulin implants on spring fertility, lactation and down of cashmere does. Proc. N. Z. Soc. Anim. Prod. 49, 39-41.

O'Neill, K.T., Litherland, A.J. \& Hamilton, G., 1992. Melatonin for cashmere production in breeding does. Proc. N. Z. Soc. Anim. Prod. 52, 161-164.

Santiago-Moreno, J., López-Sebastián, A., Del Campo, A., González-Bulnes, A., Picazo, R. \& Gómez-Brunet, A., 2004. Effect of constant-release melatonin implants and prolonged exposure to a long day photoperiod on prolactin secretion and hair growth in mouflon (Ovis gmelini musimon). Domest. Anim. Endocrinol. 26, 303-314.

SAS, 2009. Statistical Analysis Systems, OnlineDoc®, Version 9.2, SAS Institute Inc., Cary, NC. USA.

Wang, L.F., Lu, D.X., Sun, H.Z., Zhao, X.Y. \& Shan, D., 2006. Effects of photoperiod and melatonin on nitrogen partitioning and cashmere growth in Inner Mongolia White Cashmere goats. Sci. Agric. Sin. 39, 1004-1010 (in Chinese).

Wuliji, T., Litherland, A., Goetsch, A.L., Sahlu, T., Puchala, R., Dawson, L.J. \& Gipson, T., 2003. Evaluation of melatonin and bromocryptine administration in Spanish goats: II. Effect on seasonal cashmere growth, yield and fiber characteristics of does. Small Rumin. Res. 49, 41-49.

Yue, C.W., Zhang, W., Kong, X.H., Liu, H.Y. \& Jia, Z.H., 2007. Effect of melatonin on cashmere performance in Inner Mongolia white cashmere goats. Chin. J. Anim. Sci. 43, 32-34. (in Chinese, English abstract).

Zhang, W., 2011. Study on the fur growth rules of Inner Mongolia cashmere goats. M.Sc. thesis, Inner Mongolia Agricultural University, Hothot, Inner Mongolia, China. (in Chinese, English abstract).

Zarazaga, L.A., Celi, I., Guzmán, J.L. \& Malpaux, B., 2011. The effect of nutrition on the neural mechanisms potentially involved in melatonin-stimulated LH secretion in female Mediterranean goats. J. Endocrinol. 211, 263-272. 